\title{
Mass and Spatial Distributions of Subhaloes in $\Lambda$ CDM Cosmological Simulations
}

\author{
Nurmi P. ${ }^{1}$, Heinämäki P. ${ }^{1}$, Holopainen J. ${ }^{1}$, Pihajoki P. ${ }^{1}$, Saar E. ${ }^{2}$, \\ Einasto M. ${ }^{2}$, and Einasto $\mathrm{J}^{2}$ \\ ${ }^{1}$ Tuorla Observatory, University of Turku, Väisäläntie 20, FI-21500 Piikkiö, Finland \\ email: pasnurmi@utu.fi \\ ${ }^{2}$ Tartu Observatory, Tõravere Tartumaa, 61602, Estonia
}

\begin{abstract}
We study subhalo populations surrounding massive dark matter haloes by using three AMIGA simulations each having different mass and spatial resolutions. Our analysis shows that the slope of the subhalo mass function has a value 0.9 , which agrees with previous studies. The dependence of mass functions on redshift is the same for subhaloes and main haloes. In all simulations, combined subhalo masses are about 0.1-0.2 of main halo masses and this mass fraction increases slightly with redshift and the mass of the main halo. The distribution of mass fractions for subhaloes is close to Gaussian at $z=0$ and differs slightly at earlier epochs. Spatial distribution of subhaloes as measured in units of virial radius $R_{v i r}$ of the main halo does not depend on redshift and follows $r^{1 / 3}$ rule. Spatial distribution of all haloes surrounding main haloes continues up to 3 times $R_{v i r}$ with equal slope but lower amplitude. Beyond 16 times $R_{v i r}$, the average distribution of haloes becomes uniform.
\end{abstract}

Keywords. Methods: n-body simulations, Cosmology: miscellaneous-dark matter

\section{Description of the project}

We have run three $\Lambda \mathrm{CDM}$ cosmological simulations with different resolutions and volumes: $10 \mathrm{Mpc} / \mathrm{h}, 40 \mathrm{Mpc} / \mathrm{h}$ and $80 \mathrm{Mpc} / \mathrm{h}$. Mass resolutions are $4.47 \times 10^{6} \mathrm{M}_{\odot} / \mathrm{h}$, $2.86 \times 10^{8} \mathrm{M}_{\odot} / \mathrm{h}$ and $2.29 \times 10^{9} \mathrm{M}_{\odot} / \mathrm{h}$, respectively. The code used in the calculations is the AMIGA package (previously MLAPM) developed by Knebe et al. (2001). We identified main haloes and their subhaloes using the MHF (Gill et al. 2004) specifically designed to work together with AMIGA.

The general aim of this project is to study subhalo content of the haloes, the properties of subhaloes and to investigate how the surroundings of main haloes and different properties of subhaloes are correlated with environment and formation history. In the first stage (Nurmi et al. 2006) we have analysed the distributions of mass fractions and mass functions and compared the results to earlier estimates (Gao et al. 2004).

\section{Acknowledgements}

Nurmi would like to acknowledge the Magnus Ehrnrooth foundation and Finnish Academy for the support of this work. The present study was also supported by Estonian Science Foundation grants No. 4695.

\section{References}

Gao et al. 2004, MNRAS 355, 819.

Gill et al. 2004, MNRAS 351, 399.

Knebe et al. 2001, MNRAS 325, 845.

Nurmi et al. 2006, A\&BA in preparation. 